\title{
ON THE GALOIS CORRESPONDENCE FOR HOPF GALOIS STRUCTURES
}

\author{
LINDSAY N. CHILDS
}

\begin{abstract}
We study the question of the surjectivity of the Galois correspondence from subHopf algebras to subfields given by the Fundamental Theorem of Galois Theory for abelian Hopf Galois structures on a Galois extension of fields with Galois group $\Gamma$, a finite abelian $p$-group. Applying the connection between regular subgroups of the holomorph of a finite abelian $p$-group $(G,+)$ and associative, commutative nilpotent algebra structures $A$ on $(G,+)$, we show that if $A$ gives rise to a $H$-Hopf Galois structure on $L / K$, then the $K$-subHopf algebras of $H$ correspond to the ideals of $A$. As applications, we show that if $G$ and $\Gamma$ are both elementary abelian $p$-groups, then the only Hopf Galois structure on $L / K$ of type $G$ for which the Galois correspondence is surjective is the classical Galois structure. Also, if $\Gamma$ is elementary abelian of order $p^{n}$ and $p>n$ there exists an $H$-Hopf Galois structure on $L / K$ for which there are exactly $n+1 K$-sub-Hopf algebras (but approximately $n p^{n}$ intermediate subfields for $n$ large). By contrast, if $\Gamma$ is cyclic of order $p^{n}, p$ odd, then for every Hopf Galois structure on $L / K$, the Galois correspondence is surjective.
\end{abstract}

\section{INTRODUCTION}

The Fundamental Theorem of Galois Theory (FTGT) of Chase and Sweedler [CS69] states that if $L / K$ is a $H$-Hopf Galois extension of fields for $H$ a $K$-Hopf algebra $H$, then there is an injection $\mathcal{F}$ from the set of $K$-sub-Hopf algebras of $H$ to the set of intermediate fields $K \subseteq E \subseteq L$ given by sending a $K$-subHopf algebra $J$ to $\mathcal{F}(J)=L^{J}$. The strong form of the FTGT holds if the injection is also a surjection. For a classical Galois extension of fields with Galois group $\Gamma$, the FTGT holds in its strong form. It is known from [GP87] that if $L / K$ is a (classical) Galois extension with non-abelian Galois group $\Gamma$, then there is a Hopf Galois structure on $L / K$ so that $\mathcal{F}$ maps onto the subfields $E$ of $L$ that are normal over $K$. So if $\Gamma$ is not a Hamiltonian group [Ha59, 12.5], then $L / K$ has a Hopf Galois structure for which the strong form of the FTGT does not hold. In particular, the strong

Date: May 21, 2019. 
form fails extremely for the unique [By04] non-classical Hopf Galois structure on $L / K$ when $\Gamma$ is a non-abelian simple group.

Suppose $L / K$ is a classical Galois extension with Galois group $\Gamma$. If $L / K$ is also $H$-Hopf Galois for some $K$-Hopf algebra $H$, then $L \otimes_{K} K$ is $L \otimes_{K} H$-Hopf Galois over $L$, and by [GP87], $L \otimes_{K} H \cong L N$ for some regular subgroup $N$ of $\operatorname{Perm}(G)$ normalized by $\lambda(\Gamma)$, the image in $\operatorname{Perm}(\Gamma)$ of the left regular representation of $\Gamma$ on $\Gamma$. If $G$ is an abstract group and $\alpha: G \rightarrow \operatorname{Perm}(\Gamma)$ is an injective homomorphism from $G$ to $\operatorname{Perm}(\Gamma)$ whose image $\alpha(G)=N$, then we say that $H$ has type $G$.

Implicit in [GP87], and made explicit by Crespo, Rio and Vela ([CRV16], Proposition 2.2), is that the $K$-subHopf algebras of $H$ correspond to the subgroups of $\alpha(G)$ that are normalized by $\lambda(\Gamma)$ in $\operatorname{Perm}(\Gamma)$.

Nearly all of the examples examining the success or failure of the strong form of the FTGT for a non-classical Hopf Galois structure on a classical Galois extension $L / K$ with Galois group $\Gamma$ involve nonabelian groups. Perhaps the only wholly abelian example of failure in the literature is in [CRV15], 3.1, where $\Gamma \cong C_{2} \times C_{2}$ and $L / K$ has a Hopf Galois structure by $H$, a $K$-Hopf algebra which is a $K$-form of $L C_{4}$. Then by classical Galois theory, there are three intermediate subfields between $K$ and $L$, but $L C_{4}$ has only one intermediate $L$-Hopf algebra, so $H$ can have at most one intermediate $K$-subHopf algebra. Hence the strong form of the FTGT cannot hold for that Hopf Galois structure.

Here we assume that $L / K$ is a Galois extension with Galois group an abelian $p$-group $\Gamma$ of order $p^{n}$. We look at Hopf Galois structures on $L / K$ by $K$-Hopf algebras $H$ of type $G$ where $G$ is abelian, also of order $p^{n}$. There is a sequence of correspondences associated to $H$ :

- the abelian $K$-Hopf algebra $H$ corresponds by base change and Galois descent (as in [GP87]) to the $L$-Hopf algebra $L \otimes_{K} H$, which equals $L N$ for some regular abelian subgroup $N$ of $\operatorname{Perm}(\Gamma)$ normalized by $\lambda(\Gamma)$;

- if $\alpha: G \rightarrow \operatorname{Perm}(\Gamma)$ is a regular embedding with image $N$, then $\alpha$ corresponds to a regular embedding $\beta: \Gamma \rightarrow \operatorname{Hol}(G)$, as shown in [By96];

- writing $G=(G,+)$, the regular subgroup $\beta(\Gamma)$ of $\operatorname{Hol}(G)$ corresponds to an associative, commutative nilpotent ring structure $A$ on $(G,+)$, as shown in [CDVS06] and [FCC12].

Our main result implies that under these correspondences, the $K$ sub-Hopf algebras of $H$ correspond to ideals of $A$. As a consequence, we show that for $\Gamma \cong G$ an elementary abelian $p$-group, the only case 
where the FTGT holds in its strong form is if $H$ is the classical Galois structure on $L / K$.

This paper and [FCC12], [Ch15] and [Ch16] demonstrate in different ways the usefulness of the correspondence of [CDVS06] in the Hopf Galois theory of Galois extensions of fields whose Galois group is a finite abelian $p$-group.

\section{TRAnslating TO AND FROM THE HOLOMORPH}

Let $L / K$ be a Galois extension with Galois group $\Gamma$ and let $G$ be a group of the same cardinality as $\Gamma$. The $H$-Hopf Galois structures on $L / K$ of type $G$ correspond to images of regular embeddings $\alpha: G \rightarrow$ $\operatorname{Perm}(\Gamma)$ where $\alpha(G)$ is normalized by $\lambda(G)$.

As shown in [By96], a regular embedding $\alpha: G \rightarrow \Gamma$ whose image $\alpha(G)$ is normalized by $\lambda(\Gamma)$ corresponds to a regular embedding $\beta$ : $\Gamma \rightarrow \operatorname{Hol}(G)$, where

$$
\operatorname{Hol}(G)=\rho(G) \cdot \operatorname{Aut}(G) \subset \operatorname{Perm}(G)
$$

is the normalizer of $\lambda(G)$ in $\operatorname{Perm}(G)$. Here $\rho: G \rightarrow \operatorname{Perm}(G)$ is the right regular representations of $G$ in $\operatorname{Perm}(G)$. The relationship between $\alpha$ and $\beta$ is as follows:

Let $\beta: \Gamma \rightarrow \operatorname{Hol}(G)$ be a regular embedding. Define $b: \Gamma \rightarrow G$ by

$$
b(\gamma)=\beta(\gamma)\left(e_{G}\right)
$$

for $\gamma$ in $\Gamma, g$ in $G$, where $e_{G}$ is the identity element of $G$. Then

$$
\beta(\gamma)(g)=\left(b(\lambda(\gamma)) b^{-1}\right)(g)=(C(b) \lambda(\gamma))(g)
$$

Define $\alpha: G \rightarrow \operatorname{Perm}(\Gamma)$ by

$$
\alpha(g)(\gamma)=\left(b^{-1}(\lambda(g)) b\right)(\gamma)=\left(C\left(b^{-1}\right) \lambda(g)\right)(\gamma) .
$$

The following formulas will be useful below.

Proposition 2.1. Suppose $\beta: \Gamma \rightarrow \operatorname{Hol}(\lambda(G))$ is an regular embedding, and let $\alpha=C\left(b^{-1}\right) \lambda_{G}: G \rightarrow \operatorname{Perm}(\Gamma)$ be the regular embedding corresponding to $\beta$. Then for all $\gamma$ in $\Gamma$ and $g$ in $G$, there is some $h$ in $G$ so that

$$
\beta(\gamma) \lambda(g) \beta(\gamma)^{-1}=\lambda(h)
$$

and

$$
\lambda(\gamma) \alpha(g) \lambda(\gamma)^{-1}=\alpha(h) .
$$

Proof. The first formula follows because $\beta$ maps $\Gamma$ into $\operatorname{Hol}(G)$, the normalizer of $\lambda(G)$ in $\operatorname{Perm}(G)$. Since $C\left(b^{-1}\right)(\beta)(\gamma)=\lambda(\gamma)$ and $C\left(b^{-1}\right) \lambda(g)=$ $\alpha(g)$, the second formula follows from the first by applying $C\left(b^{-1}\right)$ to the first formula. 


\section{On the Galois Correspondence for Hopf Galois STRUCTURES}

Let $L / K$ be a Galois extension of fields with Galois group $\Gamma$, and suppose $\alpha: G \rightarrow \operatorname{Perm}(\Gamma)$ is a regular embedding such that $\lambda(\Gamma)$ normalizes $\alpha(G)$. Then by descent, $H=L[\alpha(G)]^{G}$ is a $K$-Hopf algebra and there is an action of $H$ on $L$ making $L / K$ into an $H$-Hopf Galois extension. (See [GP87].) The Fundamental Theorem of Galois Theory of Chase and Sweedler [CS69] gives an injection $\mathcal{F}$ from the set of $K$ subHopf algebras of $H$ to the set of intermediate fields $F$ with $K \subseteq$ $F \subseteq L$ by

$$
H^{\prime} \mapsto L^{H^{\prime}}=\left\{x \in L \mid h x=\epsilon(h) x \text { for all } h \text { in } H^{\prime}\right\} .
$$

From Proposition 2.2 of [CRV16], the $K$-subHopf algebras of $H$ correspond to $\lambda(\Gamma)$-invariant subgroups of $\alpha(G)$. Thus to study the image of $\mathcal{F}$, we look at the $\lambda(\Gamma)$ invariant subgroups of $\alpha(G)$.

We do this for $G$ a finite abelian $p$-group by utilizing a characterization of regular subgroups of $\operatorname{Hol}(G)$ due to Caranti, Della Volta and Sala [CDVS06] as extended in Proposition 2 of [FCC12]:

Proposition 3.1. Let $(G,+)$ be a finite abelian p-group. Then each regular subgroup of $\operatorname{Hol}(G)$ is isomorphic to the group $(G, \circ)$ induced from a structure $(G,+, \cdot)$ of a commutative, associative nilpotent ring (hereafter, "nilpotent") on $(G,+)$, where the operation o is defined by $g \circ h=g+h+g \cdot h$.

The idea is the following: Let $(G,+)$ be an abelian group of order $p^{n}$, and suppose that $A=(G,+, \cdot)$ is a nilpotent ring structure on $(G,+)$ yielding the operation $\circ$. Define $\tau:(G, \circ) \rightarrow \operatorname{Hol}(G,+)$ by $\tau(g)(x)=g \circ x$. Then $\tau(g)(0)=g$, and

$$
\tau(g) \tau\left(g^{\prime}\right)(x)=\tau(g)\left(g^{\prime} \circ x\right)=g \circ\left(g^{\prime} \circ x\right)=\left(g \circ g^{\prime}\right) \circ x=\tau\left(g \circ g^{\prime}\right)(x),
$$

so $\tau$ is an isomorphism from $(G, \circ)$ into $\operatorname{Hol}(G,+)$ whose image $\tau(G, \circ)=$ $T$ is a regular subgroup of $\operatorname{Hol}(G)$. This process is reversible: given a regular subgroup $T$ of $\operatorname{Hol}(G,+)$, there is a nilpotent ring structure $(G,+, \cdot)$ on $G$, which defines the o operation as above and yields a unique isomomorphism $\tau:(G, \circ) \rightarrow T$ so that $\tau(g)(x)=g \circ x$.

Now suppose $L / K$ be a Galois extension with Galois group $\Gamma$, a finite abelian $p$-group of order $p^{n}$, and $\xi: \Gamma \rightarrow(G, \circ)$ is some isomorphism.

Given $A$ and $\tau$, let $\beta=\tau \xi: \Gamma \rightarrow T$, an embedding of $\Gamma$ onto $T$ in $\operatorname{Hol}(G)$. Then $\beta$ yields the map

$$
b: \Gamma \rightarrow G
$$


by

$$
b(\gamma)=\beta(\gamma)(0)=\tau(\xi(\gamma))(0)=\xi(\gamma) \circ 0=\xi(\gamma) .
$$

Thus $b$ is an isomorphism from $\Gamma$ to $(G, \circ)$. Then $b$ defines an embedding $\alpha:(G,+) \rightarrow \operatorname{Perm}(\Gamma)$, and we have the relations of Proposition 2.1) for all $\gamma$ in $\Gamma, g$ in $G$, there is some $h$ in $G$ so that

$$
\lambda(\gamma) \alpha(g)=\alpha(h) \lambda(\gamma)
$$

and

$$
\beta(\gamma) \lambda(g)=\lambda(h) \beta(\gamma)
$$

Theorem 3.2. Suppose the nilpotent algebra $A=(G,+, \cdot)$ yields the regular embedding $\alpha:(G,+) \rightarrow \operatorname{Perm}(\Gamma)$ whose image is normalized by $\lambda(\Gamma)$. Then the lattice (under inclusion) of $\lambda(\Gamma)$-invariant subgroups of $\alpha(G)$ is isomorphic to the lattice of ideals of $A$.

Proof. First, $\alpha: G \rightarrow \operatorname{Perm}(\Gamma)$ is an injective homomorphism from $(G,+)$ to $\operatorname{Perm}(\Gamma)$. So if $J$ is an additive subgroup of $G$, then $\alpha(J)$ is a subgroup of $\alpha(G) \subset \operatorname{Perm}(\Gamma)$. Conversely, if $J$ is a subset of $G$ and $\alpha(J)$ is a subgroup of $\alpha(G)$, then for $s, t$ in $J, \alpha(s+t)=\alpha(s) \alpha(t)$ is in $\alpha(J)$, so $J$ is an additive subgroup of $G$. So there is a bijection between subgroups of $(G,+)$ and subgroups of $\alpha(G)$. Clearly $J_{1} \subseteq J_{2}$ iff $\alpha\left(J_{1}\right) \subseteq \alpha\left(J_{2}\right)$, so the bijection is lattice-preserving.

Suppose the image $\alpha(G)$ of $\alpha$ is normalized by $\lambda(\Gamma)$, so for all $\gamma$ in $\Gamma, g$ in $G$, there is some $h$ in $G$ so that

$$
\lambda(\gamma) \alpha(g) \lambda(\gamma)^{-1}=\alpha(h) .
$$

This equation holds iff

$$
\beta(\gamma) \lambda_{G}(g)=\lambda_{G}(h) \beta(\gamma) .
$$

Recalling that $A=(G,+, \cdot)=(G, \circ)$, factor $\beta=\tau \xi$ where $\xi: \Gamma \rightarrow A=$ $(G, \circ)$ is an isomorphism and $\tau: A=(G, \circ) \rightarrow \operatorname{Hol}(G)$ an embedding. Let $\xi(\gamma)=k$ in $A$. Then the last equation is

$$
\tau(k) \lambda_{G}(g)=\lambda_{G}(h) \tau(k),
$$

and applying this to $x$ in $G$ gives

$$
\begin{gathered}
\tau(k)(g+x)=h+\tau(k)(x), \\
k \circ(g+x)=h+k \circ x .
\end{gathered}
$$

Viewing this equation in $A$ where $a \circ b=a+b+a \cdot b$, we have

$$
k+(g+x)+k \cdot g+k \cdot x=h+k+x+k \cdot x .
$$

This last equation reduces to

$$
h=g+k \cdot g .
$$


Now suppose $J$ is an ideal of $A$ and $g$ is in $J$. Then $k \cdot g$ is in $J$, so $h$ is in $J$, and so $\lambda(\gamma)$ conjugates $\alpha(g)$ in $\alpha(J)$ to an element of $\alpha(J)$. So $\alpha(J)$ is normalized by $\lambda(\Gamma)$ in $\operatorname{Perm}(\Gamma)$.

Conversely, suppose $J$ is an additive subgroup of $(G,+, \cdot)=A$ and $\alpha(J)$ is normalized by $\lambda(\Gamma)$. Then for all $\gamma$ in $G, g$ in $J$,

$$
\lambda(\gamma) \alpha(g) \lambda(\gamma)^{-1}=\alpha(h)
$$

and $\alpha(h)$ is in $\alpha(J)$. So $h$ is in $J$. Hence for all $k=\xi(\gamma)$ in $G$, and $g$ in $J, h=g+k \cdot g$ is in $J$. Now $J$ is an additive subgroup of $A$, so $k \cdot g$ is in $J$ for all $k$ in $G, g$ in $J$. Thus $J$ is an ideal of $A$.

\section{EXAmples}

Theorem 3.2 transforms the problem of describing the image of the Galois correspondence map $\mathcal{F}$ on a $H$-Hopf Galois structure on $L / K$ to the study of the ideals of the nilpotent algebra associated to $H$. In this section we look at some examples.

Theorem 4.1. Let $L / K$ be a Galois extension of fields with Galois group $\Gamma$ an elementary abelian p-group of order $p^{n}$. Suppose $L / K$ has a Hopf Galois structure by an abelian Hopf algebra $H$ of type $G$ where $G$ is an elementary abelian p-group. Let $A$ be a nilpotent ring structure yielding the regular subgroup $T \cong(G, \circ) \subset \operatorname{Hol}(G)$ corresponding to $H$, where $(G, \circ) \cong \Gamma$. Then the $H$-Hopf Galois structure on $L / K$ satisfies the strong form of the FTGT if and only if $A$ is the trivial nilpotent algebra satisfying $A^{2}=0$, if and only if $T=\rho(G)$, iff $H$ is the classical Galois structure by $K \Gamma$ ) on $L / K$.

Proof. If $A^{2}=0$, then $(G, \circ)=(G,+)$, so the regular subgroup $T$ acts on $G$ by $\tau(g)(h)=g \circ h=g+h$, hence $T=\lambda(G)$. Since $G$ is abelian, the corresponding Hopf Galois structure on $L / K$ is the classical structure by the $K$-Hopf algebra $K[\Gamma]$. So the Galois correspondence holds in its strong form.

For the converse, view $(G,+)$ as an $n$-dimensional $\mathbb{F}_{p}$-vector space. Suppose $A^{2} \neq 0$. Then for some $a, b$ in $A, a b \neq 0$. Then the subspace $\mathbb{F}_{p} a$ does not contain $a b$. For if $a b=r a$ for $r \neq 0$ in $\mathbb{F}_{p}$, then $a=s b a$ for $s \neq 0$ in $\mathbb{F}_{p}$. Then

$$
a=(s b) a=(s b)^{2} a=\ldots=(s b)^{n+1} a=0
$$

since $A$ is nilpotent of dimension $n$, hence $(s b)^{n+1}=0$. Thus the subspace $\mathbb{F}_{p} a$ is not an ideal of $A$.

The subgroup $\alpha\left(\mathbb{F}_{p} a\right)$ of $\alpha(G)$ is then not normalized by $\lambda(\Gamma)$. But $\Gamma \cong G$, so there are bijections between subgroups of $\alpha(G)$, subgroups of $G$, subgroups of $\Gamma$ and (by classical Galois theory) subfields of $L$ 
containing $K$. If some subgroup of $\alpha(G)$ is not normalized by $\lambda(\Gamma)$, then the number of $K$ - subHopf algebras of $H=L[\alpha(G)]^{G}$ is strictly smaller than the number of subfields between $K$ and $L$. So the Galois correspondence for the $H$-Hopf Galois structure on $L / K$ does not hold in its strong form.

By choosing a particular nilpotent algebra structure on $\left(\mathbb{F}_{p}^{n},+\right)$ we can see how badly the Galois correspondence can fail to be surjective.

Let $A$ be the primitive $n$-dimensional nilpotent $\mathbb{F}_{p}$-algebra generated by $z$ with $z^{n+1}=0$. Then $(A,+) \cong\left(\mathbb{F}_{p}^{n},+\right)$ and so the multiplication on $A$ yields a nilpotent $\mathbb{F}_{p}$-algebra structure on $(G,+)=\left(\mathbb{F}_{p}^{n},+\right)$. Let $G=\left(\mathbb{F}_{p}^{n}, \circ\right)$ where the operation $\circ$ is defined using the multiplication on $A$ by $a \circ b=a+b+a \cdot b$.

Theorem 4.2. Let $G$ be an elementary abelian p-group of order $p^{n}$. Let $A$ be a primitive $\mathbb{F}_{p}$-algebra structure $A$ on $G$, and let $(G, \circ)$ be the corresponding group structure on $\mathbb{F}_{p}^{n}$. Suppose $L / K$ is a Galois extension of fields with Galois group $\Gamma \cong(G, \circ)$. Then the primitive nilpotent $\mathbb{F}_{p}$-algebra $A$ corresponds to an $H$-Hopf Galois structure on $L / K$ for some $K$-Hopf algebra $H$, and the $K$-subHopf algebras of $H$ form a descending chain

$$
H=H_{1} \supset H_{2} \supset \ldots \supset H_{n} \supset K .
$$

Hence the Galois correspondence $\mathcal{F}$ for $H$ maps onto exactly $n+1$ fields $F$ with $K \subseteq F \subseteq L$.

Proof. Given Theorem 3.2, we just need to show that ideals of $A$ are $J_{i}=\left\langle z^{i}\right\rangle$ for $i=1, \ldots, n$.

Suppose $J$ is an ideal of $A$ and has an element $r\left(z^{k}+z^{k+r} b\right)$ of minimal degree $k$, where $r \neq 0$ in $\mathbb{F}_{p}, b$ in $A$. Then $J$ also contains

$$
z^{k}+z^{k+r} b
$$

and

$$
\left(z^{k}+z^{k+r} b\right)\left(-z^{r} b\right)=-z^{k+r} b-z^{k+2 r} b^{2},
$$

hence their sum,

$$
z^{k}-z^{k+2 r} b^{2}=z^{k}+z^{k+r^{\prime}} b^{\prime}
$$

where $r^{\prime}>r$. Repeating this argument until $r^{\prime}>n$ shows that $J$ contains $z^{k}$, hence $J \supseteq J_{k}=\left\langle z^{k}\right\rangle$. Since $J_{k}=\left\langle z^{k}\right\rangle$ contains every element of degree $\geq k, J=J_{k}$. Thus $A$ has exactly $n+1$ ideals. Since the correspondence between ideals of $A$ and $\lambda(\Gamma)$ invariant subgroups of $\alpha(G)$ is lattice-preserving, we have a single filtration

$$
\alpha(G)=\alpha\left(J_{1}\right) \supset \alpha\left(J_{2}\right) \supset \ldots \supset \alpha\left(J_{n}\right) \supset 0 .
$$


of $\lambda(G)$-invariant subgroups of $\alpha(G)$. If $H$ is the corresponding $K$ Hopf algebra making $L / K$ into a Hopf Galois extension, then $H$ has a unique filtration of $K$-sub-Hopf algebras,

$$
H=H_{1} \supset H_{2} \supset \ldots \supset H_{n} \supset K .
$$

For $A$ a primitive nilpotent $\mathbb{F}_{p}$-algebra with $A^{n+1}=0$, the corresponding group $(G, \circ)$ is isomorphic (by $a \mapsto 1+a$ ) to the group of principal units of the truncated polynomial ring $\mathbb{F}_{p}[x] /\left(x^{n+1} \mathbb{F}_{p}[x]\right.$. The structure of that group is described in Corollary 3 of [Ch07]. In particular $(G, \circ)$, hence $\Gamma$, is an elementary abelian $p$-group if and only if $p>n$.

In Theorem 4.2, when $p>n$, then $L / K$ is classically Galois with Galois group $\Gamma \cong\left(\mathbb{F}_{p}^{n},+\right)$. So the number of subgroups of $\Gamma$, and hence the the number of subfields $E$ with $K \subseteq E \subseteq L$, is equal to the number of subspaces of $\mathbb{F}_{p}^{n}$, namely

$$
\sum_{r=1}^{n} \frac{\left(p^{n}-1\right)\left(p^{n}-p\right) \cdots\left(p^{n}-p^{r-1}\right)}{\left(p^{r}-1\right)\left(p^{r}-p\right) \cdots\left(p^{r}-p^{r-1}\right)} \sim n p^{n}
$$

for $n$ large. So the Galois correspondence map $\mathcal{F}$ is extremely far from being surjective for a Hopf Galois structure corresponding to a nilpotent algebra structure $A$ with $\operatorname{dim}\left(A / A^{2}\right)=1$.

By contrast,

Proposition 4.3. Let $L / K$ be a Galois extension of fields with Galois group $\Gamma$ cyclic of order $p^{n}, p$ odd. Let the $K$-Hopf algebra $H$ give a Hopf Galois structure on $L / K$. Then $H$ has type $G$ where $G \cong \Gamma$, and the Galois correspondence for that Hopf Galois structure holds in its strong form.

Proof. From [Ko98] it is known that if $\Gamma$ is cyclic of order $p^{n}$ then every Hopf Galois structure must have type $G \cong \Gamma$. So let $G$ be cyclic of order $p^{n}$, which we identify with $\left(\mathbb{Z} / p^{n} \mathbb{Z},+\right)$. Then we view $\operatorname{Hol}(G)=G \rtimes \operatorname{Aut}(G)$ as the set of pairs $(a, g)$ where $a$ and $g$ are modulo $p^{n}$ and $(g, p)=1$, or equivalently as the set of matrices

$$
\left(\begin{array}{ll}
g & a \\
0 & 1
\end{array}\right)
$$

in $\operatorname{Aff}_{1}\left(\mathbb{Z} / p^{n} \mathbb{Z}\right) \subset \mathrm{GL}_{2}\left(\mathbb{Z} / p^{n} \mathbb{Z}\right)$, acting on $s$ in $G$ by

$$
\left(\begin{array}{ll}
g & a \\
0 & 1
\end{array}\right)\left(\begin{array}{l}
s \\
1
\end{array}\right)=\left(\begin{array}{c}
g s+a \\
1
\end{array}\right) \text {. }
$$


View $\Gamma$ as the free $\mathbb{Z} / p^{n} \mathbb{Z}$-module with basis $y$. From Proposition 2 of [Ch11], the $p^{n-1}$ regular embeddings $\beta: \Gamma=\left(\mathbb{Z} / p^{n} \mathbb{Z}\right) y \rightarrow \operatorname{Hol}(G)$ are determined by $\beta(y)$ where

$$
\beta(y)=\left(\begin{array}{cc}
1+p d & -1 \\
0 & 1
\end{array}\right)
$$

for some $d$ modulo $p^{n-1}$. So in the notation of the last section,

$$
\tau(-1)=\left(\begin{array}{cc}
1+p d & -1 \\
0 & 1
\end{array}\right)
$$

and acts on $s$ in $G$ as above. That action defines the operation $\circ$ on $G$ by

$$
(-1) \circ s=(1+p d) s-1=-1+s+p d s .
$$

The multiplication on $(G,+)$ to make $(G,+, \cdot)=A$ a nilpotent algebra is then defined by

$$
(-1) \cdot s=(-1) \circ s-((-1)+s)=(-1+s+p d s)+1-s=p d s .
$$

By distributivity, for every $r, s$ in $\mathbb{Z} / p^{n} \mathbb{Z}$,

$$
-r \cdot s=r s p d \text {. }
$$

Replacing $d$ by $-d$, let $A_{d}$ be the commutative nilpotent algebra structure on $\left(\mathbb{Z} / p^{n} \mathbb{Z},+\right)$ with multiplication

$$
r \cdot s=r s p d
$$

for all $r, s$ in $\mathbb{Z} / p^{n} \mathbb{Z}$. It is then easy to check that the ideals of $A_{d}$ are the principal ideals generated by $p^{r}$, for $r=0, \ldots, n$. Since those are also the additive subgroups of $\left(A_{d},+\right)=\left(\mathbb{Z} / p^{n} \mathbb{Z},+\right)$, it follows by Theorem 3.2 that for every Hopf Galois structure on $L / K$, the Galois correspondence holds in its strong form.

\section{REFERENCES}

[By96] N. P. Byott, Uniqueness of Hopf Galois structure of separable field extensions, Comm. Algebra 24 (1996), 3217-3228.

[By04] N. P. Byott, Hopf-Galois structures on field extensions with simple Galois groups, Bull. London Math. Soc. 36 (2004), 23-29.

[CDVS06] A. Caranti, F. Dalla Volta, M. Sala, Abelian regular subgroups of the affine group and radical rings. Publ. Math. Debrecen 69 (2006), 297-308.

[CS69] S. U. Chase, M. E. Sweedler, Hopf Algebras and Galois Theory, Springer LNM 97 (1969).

[Ch89] L. N. Childs, On the Hopf Galois theory for separable field extensions, Comm. Algebra 17 (1989), 809-825.

[Ch07] L. N. Childs, Some Hopf Galois structures arising from elementary abelian p-groups, Proc. Amer. Math. Soc. 135 (2007), 3453-3460. 
[Ch11] L. N. Childs, Hopf Galois structures on Kummer extensions of prime power degree, New York J. Math. 17 (2011). 5174.

[Ch15] L. N. Childs, On Abelian Hopf Galois structures and finite commutative nilpotent rings, New York J. Math., 21 (2015), 205-229.

[Ch16] L. N. Childs, Obtaining abelian Hopf Galois structures from finite commutative nilpotent rings, arxiv: 1604.05269

[CRV15] T. Crespo, A. Rio, M. Vela, From Galois to Hopf Galois: theory and practice, Contemp. Math. 649 (2015), 29-46.

[CRV16] T. Crespo, A. Rio, M. Vela, On the Galois correspondence theorem in separable Hopf Galois theory, Pub. Mat. Debrecen 60 (2016), 221234.

[FCC12] S. C. Featherstonhaugh, A. Caranti, L. N. Childs, Abelian Hopf Galois structures on prime-power Galois field extensions, Trans. Amer. Math. Soc. 364 (2012), 3675-3684.

[GP87] C. Greither, B. Pareigis, Hopf Galois theory for separable field extensions, J. Algebra 106 (1987), 239-258.

[Ha59] M. Hall, The Theory of Groups, Macmillan, New York, 1959.

[Ko98] T.Kohl, Classification of the Hopf Galois structures on prime power radical extensions, J. Algebra 207 (1998), 525-546.

Department of Mathematics and Statistics, University at Albany, Albany, NY 12222

E-mail address: lchilds@albany.edu 